\title{
Iterative Successive Interference Cancellation for Quasi-Synchronous Block Spread CDMA Based on the Orders of the Times of Arrival
}

\author{
Yue Wang, ${ }^{1}$ Mohammud Z. Bocus, ${ }^{2}$ and Justin P. Coon ${ }^{1}$ \\ ${ }^{1}$ Telecommunication Research Laboratory (TRL), Toshiba Research Europe Limited, 32 Queen Square, Bristol BS1 4ND, UK \\ ${ }^{2}$ Centre for Communication Research, University of Bristol, Bristol BS8 1TW, UK \\ Correspondence should be addressed to Yue Wang, yue.wang@toshiba-trel.com
}

Received 31 March 2010; Revised 28 September 2010; Accepted 30 November 2010

Academic Editor: Hikmet Sari

Copyright () 2011 Yue Wang et al. This is an open access article distributed under the Creative Commons Attribution License, which permits unrestricted use, distribution, and reproduction in any medium, provided the original work is properly cited.

\begin{abstract}
Recently, a block spreading code division multiple access (BS-CDMA) technique was presented, whereby user-specific precoding along with orthogonal spreading codes is used to achieve multiuser interference- (MUI-) free reception when all users arrive at the base station simultaneously. In practice, however, imperfect synchronization destroys the orthogonality among users, and MUI occurs. To mitigate the MUI in BS-CDMA due to quasisynchronous reception, this paper proposes an iterative successive interference cancellation (SIC) receiver, where cancellation of interfering signals is ordered according to the times of arrival (TOA) of the signals from different users. The ordering criterion is justified through analysis and simulation on the average signal-tointerference-plus-noise ratio (SINR) of different users, where it is shown that in a quasisynchronous BS-CDMA system, ordering with regard to increasing TOA is equivalent to ordering with respect to decreasing average SINR, when practical channels such as the exponentially decaying channel is considered. The proposed SIC receiver is shown to achieve a performance close to a system with synchronous reception for only two iterations. In addition, an algorithm to determine the detection order of different blocks is proposed such that parallel detection of the signals from different users with reduced latency can be achieved.
\end{abstract}

\section{Introduction}

Code division multiple access (CDMA) is a popular multiple access technique that is used to support multiple users simultaneously in a network. Recently, a novel block spread CDMA (BS-CDMA) [1] framework was presented, whereby the use of user-specific, channel-independent precoding along with orthogonal spreading codes leads to variants of well-known practical multiple access techniques such as orthogonal frequency division multiple access (OFDMA), which has been considered as a mandatory technology in the Long Term Evolution (LTE) standard [2]. In a BS-CDMA system, the orthogonality among users guarantees multiuser interference (MUI) free reception when perfect synchronization among users is achieved. In practice, however, perfect synchronization for signals from different users is usually hard to obtain due to different signal propagation delays [3], resulting in MUI. Consequently, conventional receivers for synchronous BS-CDMA fail to work in asynchronous systems. In fact, it was shown in [4] that even in a quasisynchronous environment where the beginnings of the signals from different users are synchronized to within a few chips, severe performance degradation due to MUI can occur.

Advanced equalizer designs were proposed to mitigate the MUI due to quasi-synchronous reception in a BSCDMA system in [4]. It was shown in [4] that, although the proposed equalizers can lower the error floor caused by MUI, a performance degradation compared to an ideal synchronous system still exists, even when error correcting codes are applied. Successive interference cancellation (SIC) is an effective MUI mitigation technique that has received considerable attention in conventional multiple access systems such as direct sequence code division multiple access (DS-CDMA) systems [5-14]. However, a BS-CDMA system is different from a DS-CDMA system in the sense that while 
a DS-CDMA system spreads each symbol from a particular user by using a user-specific spreading code, a BS-CDMA system spreads a block of precoded symbols with such a spreading code. As a result, interference cancellation schemes that work well for DS-CDMA may not be applied to BSCDMA systems. This poses new problems for BS-CDMA systems that must be addressed in practice.

For conventional DS-CDMA systems, a typical way of employing SIC is to detect the signals from each user in the order of decreasing received powers [5]. Such an SIC receiver requires disparity in the receiver power distribution among users to achieve improved performance compared to conventional receivers $[7,8,14]$. For systems with perfect power control when all reverse link signals are received at the same power level [15], SIC detection in the order of decreasing received power becomes less effective [7]. This issue is also explained in [16] from an information theoretic point of view, where it was shown that strong interference can be cancelled and that weak interference can be treated as noise without causing a significant penalty to the users rates. Equal strength interferers are more problematic. Consequently, we consider uplink BS-CDMA with perfect power control in this paper and propose a novel SIC scheme to reduce the MUI due to quasi-synchronous reception.

Apart from ordering by decreasing received powers, other ordering criteria can be used in SIC to detect the signals from different users. Ideally, one would detect the signals on an order of decreased average signal-to-interferenceplus-noise ratio (SINR) such that the first detected signals are the most reliable. Although average SINR is usually tractable through analysis, the measurement of average SINR in practice is more involved. For example, SINR values have to be measured within a finite time duration; therefore, they are sensitive to instantaneous variations in channel quality, while the mitigation of such variations requires an average of the short-term metrics over a long time duration [17]. Thanks to the special features of BS-CDMA where it has been shown in recent studies that, under certain practical circumstances, unequal MUI power may occur to different users, thus facilitating the use of other ordering criteria in an SIC receiver. For example, based on the fact that users with high mobility cause more interference to other users compared to those with low mobility in nonstationary channels, SIC by using the mobility condition to determining the order of detection was proposed in [18].

In this paper, we consider a quasi-synchronous BSCDMA system and show through analysis and simulation that for practical channels such as the exponentially decaying channel, detection with an order of increasing times of arrival (TOA) is essentially the same as an order of decreasing average SINR. Although this property of ordering holds only for particular channel models with an exponentially decaying power delay profile, it does not diminish the practicality of the proposed SIC receiver, because these channel models are considered to be in good agreement with practical channel measurements [19] and have been adopted by the 3rd Generation Partnership Project (3GPP) to model channels for cellular networks [20]. Based on a thorough analysis of the SINR in quasi-synchronous BSCDMA receiver, we propose a novel SIC receiver where the signals are detected according to an increasing order of TOA. Since TOA estimation is considered as one of the essential methods to meet the mandates on cellular operators by the Federal Communications Committee [21] (examples of TOA estimation method can be found in [22]), it is feasible to design SIC by using TOA to determine the order of detection. Note that the equivalence between ordering according to TOA and ordering according to average SINR is particular to BS-CDMA systems and does not necessarily hold for conventional asynchronous DS-CDMA systems. Therefore, ordering according to TOA is not necessarily beneficial in those systems. In fact, although SIC or multistage/iterative SIC schemes for conventional asynchronous CDMA systems have been investigated extensively in the literature (see, e.g., $[12,13,23])$, to the best of our knowledge, none of these SIC methods considered the benefit of using TOA as an ordering criterion. In addition to proposing an SIC receiver with ordering based on TOA, we also detail a low-latency algorithm for determining the order of detection for blocks from different users.

The rest of the paper is organized as follows. In Section 2, the system model of quasi-synchronous BS-CDMA is presented. In Section 3, the average SINR of a quasisynchronous BS-CDMA system is derived, where it is shown that in practical exponentially decaying channels, average SINRs of different users decrease with increasing TOA. An iterative SIC scheme based on ordering according to the TOA of the signals from different users is proposed in Section 4. Simulation results are shown in Section 5, and Section 6 concludes the paper.

\section{System Model}

Figure 1 shows the block diagram of the quasi-synchronous BS-CDMA system. Consider a BS-CDMA system with $M$ users. At the transmitter of the $\mu$ th user, information bits are encoded, interleaved, and mapped to constellation symbols, which are then arranged into blocks of $P$ symbols, with the $i$ th block of symbols for the $\mu$ th user given by a length- $P$ column vector $\mathbf{s}_{\mu}(i)$. Each block of symbols is then precoded with a $P \times P$ user-specific precoding matrix $\Lambda_{\mu}$ and subsequently block spread by a length- $M$ spreading code $\mathbf{c}_{\mu}$. In this paper, we consider the case as in [1], where discrete Fourier transform (DFT) codes are used as the spreading codes, and the precoding matrix for the $\mu$ th user is given by a diagonal matrix with its $p$ th diagonal entry being $\exp (-j 2 \pi p(m-1) / M P)$, for $p=1, \ldots, P$.

The signal of the $\mu$ th user in the $i$ th block after block spreading and precoding is given by

$$
\mathbf{x}_{\mu}(i)=\left(\mathbf{c}_{\mu} \otimes \Lambda_{\mu}\right) \mathbf{s}_{\mu}(i)
$$

where $\otimes$ denotes Kronecker product, and $\mathbf{x}_{\mu}(i)$ contains $M P$ chips.

A cyclic prefix (CP) of length $L_{\mathrm{CP}}$, at least equal to the memory order of the channel impulse response (CIR), is added at the beginning of $\mathbf{x}_{\mu}(i)$. Cyclically extended signals 


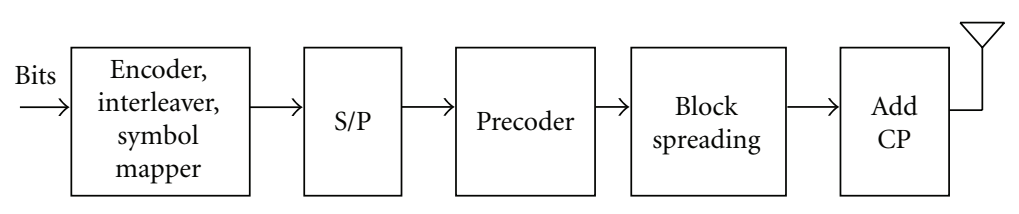

(a) Transmitter

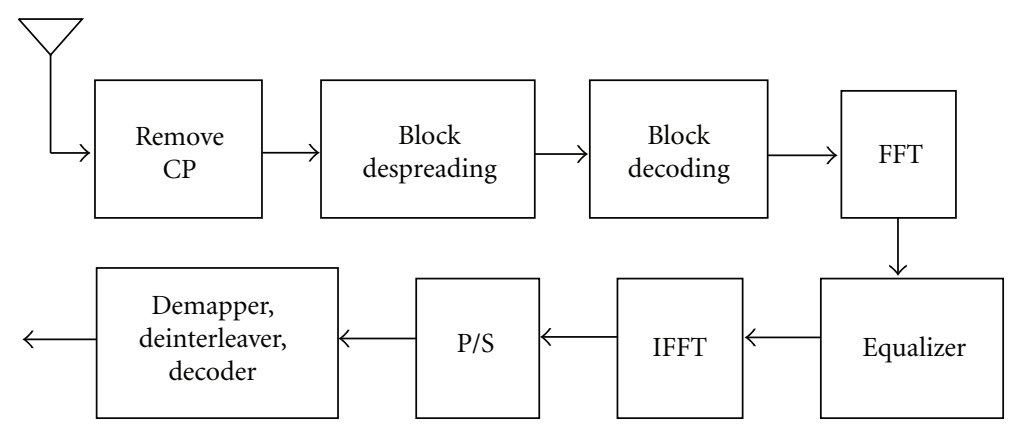

(b) Receiver

Figure 1: Transceiver of a synchronous BS-CDMA system.

of each user then go through the channel. We consider a slow time-varying channel where the CIRs in different blocks within one frame of the transmitted data are the same. For simplicity, we also assume that the CIRs for different users are of the same length $L$. The CIR of the $\mu$ th user is given by $\mathbf{h}_{\mu}=\left[h_{\mu}(0), \ldots, h_{\mu}(L-1)\right]^{T}$, where $[\cdot]^{T}$ denotes matrix transpose.

Assume that the TOA of each user is known at the base station, and the users are ordered and indexed according to their TOA. For example, the user whose signal arrives first is the first user in the ordering, and the user whose signal arrives last is the $M$ th user in the ordering. We consider chiplevel synchronization where the beginning of the signal of the $\mu$ th user arrives $\tau_{\mu}$ chips later than that of the first user, where $\left\{\tau_{\mu}\right\}$ are positive integers for $\mu \neq 1$, and $\tau_{1}=0$. Note that in some practical cases, synchronization is reasonably good such that the delays can be smaller than a chip interval. In such a case, the delays can be modeled as fractional numbers rather than integers. The analysis we present in this paper can be extended to the cases where delays are fractional by considering an oversampled system. Here, we use integer delays in the derivations and simulations for simplicity. Denote the time difference between the beginning of the signal from the $\mu$ th user and that of the $m$ th user as $\tau_{\mu \rightarrow m}=$ $\left|\tau_{\mu}-\tau_{m}\right|$. To detect the $m$ th user's message, the receiver synchronizes to the beginning of the signal of this user. We refer to the user to which the receiver is synchronized as the reference user, and the beginning of the signal of the reference user is termed the synchronization instant. Furthermore, we consider quasi-synchronous BS-CDMA where the delays are reasonably small such that $\max \left\{\tau_{\mu}\right\} \leq L \leq L_{\mathrm{CP}} \ll M P$, for all $\mu=1, \ldots, M$.

At the receiver, the $\mathrm{CP}$ is first removed from the beginning of the composite received signal. Note that to detect the signals for a given user, the receiver synchronizes to the beginning of the signals of that user and removes $L_{\mathrm{CP}}$ symbols relative to the synchronization instant. Denote the $i$ th block of the received signal after $\mathrm{CP}$ removal at the base station as $\mathbf{r}(i)$. A block despreading and decoding operation is then employed to detect the signals for each user. The signal after despreading and decoding (note that the term decoding is used here to follow the convention of [1]. The decoding operation here refers to the inverse operation of the precoding operation, which is different from the terminologies used for error correcting codes) is given by

$$
\mathbf{z}_{m}(i)=\mathbf{D}_{m}^{H} \mathbf{r}(i)
$$

where $(\cdot)^{H}$ denotes Hermitian transpose, $\mathbf{D}_{m}=\mathbf{c}_{m} \otimes \boldsymbol{\Gamma}_{m}$ is the despreading and decoding matrix for the $m$ th user, and the decoding matrix $\boldsymbol{\Gamma}_{m}$ is identical to the precoding matrix $\boldsymbol{\Lambda}_{m}$. It was shown in [4] that at a BS-CDMA receiver, the $i$ th received block of the $m$ th user after block despreading and decoding is given by

$$
\mathbf{z}_{m}(i)=M \widetilde{\tilde{\mathbf{H}}}_{m} \mathbf{s}_{m}(i)+\sum_{b=1}^{m-1} \boldsymbol{\theta}_{b \rightarrow m}+\sum_{a=m+1}^{M} \phi_{a \rightarrow m}+\mathbf{v}(i),
$$

where $M \tilde{\overline{\mathbf{H}}}_{m} \mathbf{s}_{m}(i)$ is the $i$ th received, despread block for the $m$ th user before equalization, and $\widetilde{\mathbf{H}}_{m}$ is a $P \times P$ circulant matrix with its first column being $\mathbf{h}_{m}$ appended by zeros. In addition, $\mathbf{v}(i)=\mathbf{D}_{m}^{H} \mathbf{n}(i)$ is the equivalent noise term, with $\mathbf{n}(i)$ being the white Gaussian noise vector, each entry of which having a mean of zero and a variance of $\sigma_{n}^{2}$. In addition, the second and third summation terms account for MUI, where $\phi_{a \rightarrow m}$ is the interference term from the ath user whose signal arrives later than the synchronization instant, and $\boldsymbol{\theta}_{b \rightarrow m}$ is the interference term from the $b$ th user whose signal arrives earlier than the synchronization instant, which are given by [4]

$$
\begin{aligned}
& \phi_{a \rightarrow m}=\mathbf{D}_{m}^{H}\left[\Delta_{a \rightarrow m}^{U} \mathbf{x}_{a}(i-1)-\Delta_{a \rightarrow m}^{U} \mathcal{C}_{d}^{L_{\mathrm{CP}}} \mathbf{x}_{a}(i)\right], \\
& \boldsymbol{\theta}_{b \rightarrow m}=\mathbf{D}_{m}^{H}\left[\boldsymbol{\Delta}_{b \rightarrow m}^{L} \mathbf{x}_{b}(i)-\Delta_{b \rightarrow m}^{L} \mathcal{C}_{d}^{L_{\mathrm{CP}}} \mathbf{x}_{b}(i+1)\right] .
\end{aligned}
$$


In (4) and (5), $\Delta_{a \rightarrow m}^{U}$ is an $M P \times M P$ upper triangular Toeplitz matrix with its first row being $\left[0, \ldots, 0, h_{a}(L-1)\right.$, $\left.\ldots, h_{a}\left(L-l_{a}\right)\right]$ where $l_{a}=L-L_{\mathrm{CP}}+\tau_{a \rightarrow m}-1$ for $L_{\mathrm{CP}}<$ $L+\tau_{a \rightarrow m}-1, \Delta_{b \rightarrow m}^{L}$ is an $M P \times M P$ lower triangular Toeplitz matrix with the first column being $\left[0, \ldots, 0,-h_{b}(0), \ldots,-h_{b}\left(\tau_{b \rightarrow m}-1\right)\right]^{T}$, and $\mathcal{C}_{d}^{L_{\mathrm{CP}}}$ is a circulant matrix obtained by circularly shifting the $M P \times$ $M P$ identity matrix down by $L_{\mathrm{CP}}$. Note that MUI due to quasi-synchronous reception can be reduced by using an increasing length of CP. In fact, it was shown in [4] that when $L_{\mathrm{cp}}$ is sufficiently long such that $L_{\mathrm{CP}} \geq L+\tau_{a \rightarrow m}-1$, interference due to users whose signals arrive later than the synchronization instant can be eliminated. But this requires a redundancy of at least $\tau_{a \rightarrow m}$ in the transmitted signal. Despite the length of the CP used, the interference due to users whose signals arrive earlier than the synchronization instant cannot be eliminated. In such a case, interference cancellation needs to be employed to cope with the MUI due to quasi-synchronous reception. In the following, we consider the general case where interference from both users whose signals arrive earlier or later than the synchronization instant exists.

After the despreading and decoding operation, for synchronous BS-CDMA where the interference terms are zero vectors, due to the circularity of the equivalent channel matrix $\widetilde{\mathbf{H}}_{m}$, the received signal can be detected by using a lowcomplexity frequency domain equalizer, where the received signal can be passed through a fast Fourier transform (FFT), followed by a frequency domain equalizer, and finally an inverse FFT (IFFT) to recover the message for the $m$ th user. When quasi-synchronous BS-CDMA is considered, an iterative SIC operation can be employed before the FFT to mitigate the MUI. Denote the ith signal block after SIC as $\mathbf{w}_{m}(i)$. The estimated $i$ th transmit block for the $m$ th user is given by

$$
\widehat{\mathbf{s}}_{m}(i)=\mathbf{F}^{H} \mathbf{G}_{m} \mathbf{F} \mathbf{w}_{m}(i),
$$

where $\mathbf{F}$ is the FFT matrix, and $\mathbf{G}_{m}$ is the frequency domain equalizer for the $m$ th user, which can be a zero-forcing (ZF) or linear minimum mean squared (LMMSE) equalizer. The expressions for these equalizers can be found in [24, 25]. The equalized time domain signals are then detected according to the log-likelihood criterion, given by

$$
\overline{\mathbf{s}}_{m}(i)=\arg \min _{\hat{\mathbf{s}}_{m}(i)}\left\|\hat{\mathbf{s}}_{m}(i)-\boldsymbol{\xi}\right\|^{2},
$$

where $\overline{\mathbf{s}}_{m}(i)$ is the detected $i$ th block of symbols for the $m$ th user, and $\|\cdot\|$ represents the $l_{2}$ norm operation. In addition, $\xi$ is a column vector with each element of which belongs to a set $\&$ containing the normalized constellation symbols for a given modulation. For example, $\delta=\{ \pm 1 / \sqrt{2} \pm j / \sqrt{2}\}$ when QPSK modulation is considered. The detected symbols are then demapped, deinterleaved, and decoded to recover the transmitted bits of the desired user.

\section{SINR Analysis}

We analyze the average SINR of quasi-synchronous BSCDMA in this section and show that when practical channels such as the exponentially decaying channel is considered, ordering with decreasing average SINR is equivalent to ordering with increasing TOA.

Following (3), the average SINR of quasi-synchronous BS-CDMA is given by

$$
\mathrm{SINR}_{m}=\frac{P_{s_{m}}}{P_{I_{a}}+P_{I_{b}}+\sigma_{v}^{2}},
$$

where

$$
P_{s_{m}}=\operatorname{Tr}\left\{M^{2} \mathbb{E}\left[\tilde{\mathbf{H}}_{m} \mathbf{s}_{m}^{i}\left(\mathbf{s}_{m}^{i}\right)^{H} \tilde{\mathbf{H}}_{m}^{H}\right]\right\}
$$

is the signal power

$$
\begin{aligned}
& P_{I_{a}}=\operatorname{Tr}\left\{\mathbb{E}\left[\left(\sum_{a=m+1}^{M} \boldsymbol{\phi}_{a \rightarrow m}\right)\left(\sum_{a^{\prime}=m+1}^{M} \boldsymbol{\phi}_{a^{\prime} \rightarrow m}\right)^{H}\right]\right\}, \\
& P_{I_{b}}=\operatorname{Tr}\left\{\mathbb{E}\left[\left(\sum_{b=1}^{m-1} \boldsymbol{\theta}_{b \rightarrow m}\right)\left(\sum_{b^{\prime}=1}^{m-1} \boldsymbol{\phi}_{b^{\prime} \rightarrow m}\right)^{H}\right]\right\}
\end{aligned}
$$

are the interference power from users whose signals arrive later and earlier than the $m$ th user, respectively, and

$$
\sigma_{v}^{2}=\operatorname{Tr}\left\{\mathbf{D}_{m}^{H} \mathbb{E}\left[\mathbf{n} \mathbf{n}^{H}\right] \mathbf{D}_{m}\right\}=M P \sigma_{n}^{2}
$$

is the equivalent noise power, where the second equality is obtained by using the facts that $\operatorname{Tr}\{\mathbf{A B C}\}=\operatorname{Tr}\{\mathbf{B C A}\}$ and $\mathbf{D}_{m}^{H} \mathbf{D}_{m}=M \mathbf{I}_{P}$, with $\mathbf{I}_{P}$ being the $P \times P$ identity matrix. In $(9)-(11), \mathbb{E}[\cdot]$ denotes the expectation operation and $\operatorname{Tr}\{\cdot\}$ denotes the trace of a matrix.

Assume that the transmitted signals from different users are independent, those from a given user are independent from block to block, and those within one block $\mathbf{s}_{m}^{i}$ are also independent. (Note that when error correcting coding is applied to the transmitted signals, signals within a same block may not be independent. However, the assumption of the independent signals within a block does not affect the analysis results as long as ideal (or nearly ideal) interleavers are used at the transmitted. This has been verified through simulations.) We assume each symbol has a mean of zero and a variance of $\sigma_{s}^{2}$, that is, $\mathbb{E}\left[\mathbf{s}_{m}(i)\left(\mathbf{s}_{m}(i)\right)^{H}\right]=\sigma_{s}^{2} \mathbf{I}_{P}$. Equations (9), (10) can therefore be simplified to yield

$$
\begin{gathered}
P_{s_{m}}=M^{2} \sigma_{s}^{2} \operatorname{Tr}\left\{\mathbb{E}\left[\widetilde{\mathbf{H}}_{m} \tilde{\mathbf{H}}_{m}^{H}\right]\right\}, \\
P_{I_{a}}=\operatorname{Tr}\left\{\sum_{a=m+1}^{M} \mathbb{E}\left[\boldsymbol{\phi}_{a \rightarrow m} \boldsymbol{\phi}_{a \rightarrow m}^{H}\right]\right\}, \\
P_{I_{b}}=\operatorname{Tr}\left\{\sum_{b=1}^{m-1} \mathbb{E}\left[\boldsymbol{\theta}_{b \rightarrow m} \boldsymbol{\theta}_{b \rightarrow m}^{H}\right]\right\} .
\end{gathered}
$$

It is known that $\widetilde{\mathbf{H}}_{m}$ can be decomposed as $\widetilde{\mathbf{H}}_{m}=\mathbf{F}^{H} \boldsymbol{\Xi}_{m} \mathbf{F}$, where $\boldsymbol{\Xi}_{m}$ is the diagonal matrix containing the $k$ th frequency domain channel coefficient $H_{m}(k)$ as its $k$ th diagonal entry [26]. Applying the decomposition of $\tilde{\mathbf{H}}_{m}$ to (12), we have

$$
P_{s_{m}}=M^{2} P \sigma_{s}^{2} \mathbb{E}\left[\sum_{l=0}^{L}\left|h_{m}(l)\right|^{2}\right],
$$


where the equality $\sum_{k=0}^{P-1}\left|H_{m}(k)\right|^{2}=P \sum_{l=0}^{L}\left|h_{m}(l)\right|^{2}$ is applied due to Parseval's theorem.

We now analyze the interference power due to quasisynchronous reception. Following (4) and (5), applying (1) and the property of the Kronecker product where $(\mathbf{A} \otimes$ $\mathbf{B})(\mathbf{C} \otimes \mathbf{D})=\mathbf{A B} \otimes \mathbf{C D}$, and taking the expectation over the transmitted symbols, we have

$$
\begin{aligned}
\mathbb{E}[ & \left.\phi_{a \rightarrow m} \boldsymbol{\phi}_{a \rightarrow m}^{H}\right] \\
=\mathbb{E} & {\left[\sigma_{s}^{2} \mathbf{D}_{m}^{H} \Delta_{a \rightarrow m}^{U}\left(\mathbf{c}_{a} \mathbf{c}_{a}^{H} \otimes \mathbf{I}_{P}\right)\left(\Delta_{a \rightarrow m}^{U}\right)^{H} \mathbf{D}_{m}\right.} \\
& \left.\quad+\sigma_{s}^{2} \mathbf{D}_{m}^{H} \Delta_{a \rightarrow m}^{U} \mathcal{C}_{L_{\mathrm{CP}}}^{d}\left(\mathbf{c}_{a} \mathbf{c}_{a}^{H} \otimes \mathbf{I}_{P}\right)\left(\mathcal{C}_{L_{\mathrm{CP}}}^{d}\right)^{H}\left(\Delta_{a \rightarrow m}^{U}\right)^{H} \mathbf{D}_{m}\right] \\
= & 2 \sigma_{s}^{2} \mathbb{E}\left[\mathbf{D}_{m}^{H} \Delta_{a \rightarrow m}^{U}\left(\mathbf{c}_{a} \mathbf{c}_{a}^{H} \otimes \mathbf{I}_{P}\right)\left(\Delta_{a \rightarrow m}^{U}\right)^{H} \mathbf{D}_{m}\right],
\end{aligned}
$$

where the last equality is due to the circularity of $\mathbf{c}_{a} \mathbf{c}_{a}^{H} \otimes \mathbf{I}_{P}$ when the DFT spreading codes are used, that is,

$$
\mathcal{C}_{L_{\mathrm{CP}}}^{d}\left(\mathbf{c}_{a} \mathbf{c}_{a}^{H} \otimes \mathbf{I}_{P}\right)\left(\mathcal{C}_{L_{\mathrm{CP}}}^{d}\right)^{H}=\mathbf{c}_{a} \mathbf{c}_{a}^{H} \otimes \mathbf{I}_{P} .
$$

Due to the assumption that $L_{\mathrm{CP}}<L+\tau_{a \rightarrow m}-1, \Delta_{a}^{U}$ can be decomposed into the Kronecker product

$$
\Delta_{a \rightarrow m}^{U}=\mathbf{J} \otimes \Theta_{a \rightarrow m}^{U},
$$

where $\mathbf{J}$ is a matrix obtained by shifting an $M \times M$ identity matrix to the right by $M-1$, and $\Theta_{a \rightarrow m}^{U}$ is a $P \times P$ upper triangular Toeplitz matrix with its first row being $\left[\mathbf{0}_{1 \times\left(P-l_{a}\right)}, h_{a}(L-1), \ldots, h_{a}\left(L-l_{a}\right)\right]\left(l_{a}\right.$ was previously defined as $\left.l_{a}=L-L_{\mathrm{CP}}+\tau_{a \rightarrow m}-1\right)$. Applying the decomposition of $\Delta_{a \rightarrow m}^{U}$ in (18), (16) can be rewritten as

$$
\begin{aligned}
& \mathbb{E}\left[\boldsymbol{\phi}_{a \rightarrow m} \boldsymbol{\phi}_{a \rightarrow m}^{H}\right] \\
& =2 \sigma_{s}^{2} \mathbb{E}\left[\left(\mathbf{c}_{m}^{H} \mathbf{J} \mathbf{c}_{a} \mathbf{c}_{a}^{H} \mathbf{J}^{H} \mathbf{c}_{m}\right)\left(\boldsymbol{\Gamma}_{m}^{H} \boldsymbol{\Theta}_{a \rightarrow m}^{U}\left(\Theta_{a \rightarrow m}^{U}\right)^{H} \boldsymbol{\Gamma}_{m}\right)\right] \\
& =2 \sigma_{s}^{2} \mathbb{E}\left[\boldsymbol{\Gamma}_{m}^{H} \Theta_{a \rightarrow m}^{U}\left(\boldsymbol{\Theta}_{a \rightarrow m}^{U}\right)^{H} \boldsymbol{\Gamma}_{m}\right],
\end{aligned}
$$

where the second equality is due to the fact that $\mathbf{c}_{m}^{H} \mathbf{J} \mathbf{c}_{a} \mathbf{c}_{a}^{H} \mathbf{J}^{H} \mathbf{c}_{m}=1$ when the DFT spreading codes are used. The interference power from the ath user to the reference user can therefore be rewritten as

$$
\begin{aligned}
\operatorname{Tr}\left\{\mathbb{E}\left[\phi_{a \rightarrow m} \phi_{a \rightarrow m}^{H}\right]\right\} & =2 \sigma_{s}^{2} \operatorname{Tr}\left\{\mathbb{E}\left[\Theta_{a \rightarrow m}^{H}\left(\Theta_{a \rightarrow m}^{U}\right)^{H}\right]\right\} \\
& =2 \sigma_{s}^{2} \mathbb{E}\left[\sum_{l=1}^{l_{a}} \sum_{i=1}^{l}\left|h_{a}(L-i)\right|^{2}\right],
\end{aligned}
$$

due to the fact that $\boldsymbol{\Gamma}_{m}^{H} \boldsymbol{\Gamma}_{m}=\mathbf{I}_{P}$.

Similarly, for the interference terms from users whose signals arrive later, we have

$$
\mathbb{E}\left[\boldsymbol{\theta}_{a \rightarrow m} \boldsymbol{\theta}_{a \rightarrow m}^{H}\right]=2 \sigma_{s}^{2} \mathbb{E}\left[\mathbf{D}_{m}^{H} \boldsymbol{\Delta}_{b}^{L}\left(\mathbf{c}_{b} \mathbf{c}_{b}^{H} \otimes \mathbf{I}_{P}\right)\left(\boldsymbol{\Delta}_{b}^{L}\right)^{H} \mathbf{D}_{m}\right] .
$$

Applying the decomposition of $\Delta_{b}^{L}$

$$
\Delta_{b}^{L}=\mathbf{J}^{H} \otimes \Theta_{b}^{L},
$$

where $\Theta_{b \rightarrow m}^{L}$ is a $P \times P$ lower triangular Toeplitz matrix with the first column being $\left[\mathbf{0}_{1 \times\left(P-\tau_{b-m}\right)},-h_{b}(0), \ldots,-h_{b}\left(\tau_{b \rightarrow m}-\right.\right.$ 1) $]^{T}$, we have

$$
\mathbb{E}\left[\boldsymbol{\theta}_{a \rightarrow m} \boldsymbol{\theta}_{a \rightarrow m}^{H}\right]=2 \sigma_{s}^{2} \mathbb{E}\left[\boldsymbol{\Gamma}_{m}^{H} \boldsymbol{\Theta}_{b}^{L}\left(\boldsymbol{\Theta}_{b}^{L}\right)^{H} \boldsymbol{\Gamma}_{m}\right] .
$$

The interference power from the $b$ th user to the $m$ th user is, therefore, given by

$$
\begin{aligned}
\operatorname{Tr}\left\{\mathbb{E}\left[\boldsymbol{\theta}_{a \rightarrow m} \boldsymbol{\theta}_{a \rightarrow m}^{H}\right]\right\} & =2 \sigma_{s}^{2} \operatorname{Tr}\left\{\mathbb{E}\left[\boldsymbol{\Theta}_{b \rightarrow m}^{L}\left(\boldsymbol{\Theta}_{b \rightarrow m}^{L}\right)^{H}\right]\right\} \\
& =2 \sigma_{s}^{2} \mathbb{E}\left[\sum_{l=0}^{\tau_{b-m^{-1}}} \sum_{i=0}^{l}\left|h_{b}(i)\right|^{2}\right] .
\end{aligned}
$$

Substituting (20) and (24) into (13) and (14), respectively, the interference power from users whose signals arrive later and earlier than those of the $m$ th user are given by

$$
\begin{aligned}
& P_{I_{a}}=2 \sigma_{s}^{2} \sum_{a=m+1}^{M} \mathbb{E}\left[\sum_{l=1}^{l_{a}} \sum_{i=1}^{l}\left|h_{a}(L-i)\right|^{2}\right], \\
& P_{I_{b}}=2 \sigma_{s}^{2} \sum_{b=1}^{m-1} \mathbb{E}\left[\sum_{l=0}^{\tau_{b}-m^{-1}} \sum_{i=0}^{l}\left|h_{b}(i)\right|^{2}\right] .
\end{aligned}
$$

The SINR for quasi-synchronous BS-CDMA can, therefore, be computed for given channel statistics by substituting (11), (15), (25) into (8).

Despite different channel statistics, it is noted from (25) that for channels with monotonically decreasing power delay profile, the average interference power increases with an increasing $\tau_{a \rightarrow m}$ or $\tau_{b \rightarrow m}$. As a result, ordering with decreasing average SINR is equivalent to ordering with increasing TOA for such channels. In the following, we use an example of a Rayleigh fading multipath channel with an exponentially decaying power delay profile to calculate average SINR. Such a channel model is considered to be in good agreement with practical channel measurements [19, 27] and has been adopted by the 3rd Generation Partnership Project (3GPP) to model channels for cellular networks [20].

When an exponentially decaying Rayleigh fading multipath channel is considered, the discrete channel taps are given by

$$
h_{m}(l)=\sqrt{\frac{e^{-\alpha l}}{\lambda}}\left(h_{m r}(l)+j h_{m i}(l)\right), \quad l=0, \ldots, L,
$$

where $\alpha>0$ is the decaying factor, and $\lambda$ is the normalization factor given by $\lambda=\sum_{l=0}^{L} e^{-\alpha l}$. In addition, $h_{m r}(l)$ and $h_{m i}(l)$ are the real and imaginary parts of the $l$ th channel tap for the $m$ th user, both of which are real Gaussian random variables with a mean of zero and a variance of $1 / 2$. It follows that

$$
\mathbb{E}\left[\left|h_{m}(l)\right|^{2}\right]=\frac{e^{-\alpha l}}{\lambda} .
$$


Following (15), the signal power is given by

$$
\begin{aligned}
P_{s_{m}} & =\frac{M^{2} P \sigma_{s}^{2}}{\lambda} \sum_{l=0}^{L} e^{-\alpha l} \\
& =M^{2} P \sigma_{s}^{2},
\end{aligned}
$$

which can be rewritten as a function of SNR as

$$
P_{s_{m}}=\sigma_{n}^{2} M^{2} P \cdot \mathrm{SNR},
$$

where SNR is defined as SNR $=\sigma_{s}^{2} / \sigma_{n}^{2}$. Similarly, following (25) the interference power from users whose signals arrive later and earlier than the synchronization instant are given by

$$
P_{I_{a}}=\sigma_{n}^{2} \rho_{a}(\mathrm{SNR})
$$

where

$$
\begin{aligned}
\boldsymbol{\rho}_{a}(\mathrm{SNR})= & \sum_{a=m+1}^{M} \frac{2}{\lambda\left(1-e^{-\alpha}\right)^{2}} \\
& \times\left(e^{-\alpha\left(L-l_{a}\right)}-l_{a} e^{-\alpha L}\left(1-e^{-\alpha}\right)-e^{-\alpha L}\right) \cdot \mathrm{SNR}, \\
P_{I_{b}}= & \sigma_{n}^{2} \boldsymbol{\rho}_{b}(\mathrm{SNR}),
\end{aligned}
$$

where

$$
\begin{aligned}
\boldsymbol{\rho}_{b}(\mathrm{SNR})= & \sum_{b=1}^{m-1} \frac{2}{\lambda\left(1-e^{-\alpha}\right)^{2}} \\
& \times\left(\tau_{b \rightarrow m}-\tau_{b \rightarrow m} e^{-\alpha}-e^{-\alpha}+e^{-\alpha\left(\tau_{b \rightarrow m}+1\right)}\right) \cdot \mathrm{SNR},
\end{aligned}
$$

respectively. Substituting (29)-(32) and (11) to (8), the SINR for each user as a function of SNR can be obtained as

$$
\operatorname{SINR}(\mathrm{SNR})=\frac{M^{2} P}{\boldsymbol{\rho}_{a}(\mathrm{SNR})+\boldsymbol{\rho}_{b}(\mathrm{SNR})+M P} \cdot \mathrm{SNR} .
$$

In the following, we use (33) to calculate the average SINR by considering an example BS-CDMA system with 8 users for the following three different asynchronous scenarios:

(1) signals from all users have the same TOA except one, who has a delay of 7 , that is, $\boldsymbol{\tau}=[0,0,0,0,0,0,0,7]$,

(2) signals from all users except the first user have one chip delay relative to its previous user, that is, $\tau=$ $[0,1,2,3,4,5,6,7]$,

(3) signals from the last 7 users have the same delays of 7 , that is, $\boldsymbol{\tau}=[0,7,7,7,7,7,7,7]$.

The calculated SINRs according to the three asynchronous scenarios are plotted in Figures 2, 3, and 4 and compared with the simulation results. In both simulation and analysis, we considered Rayleigh fading channels with an exponentially decaying profile. The decay factor is approximately 0.86

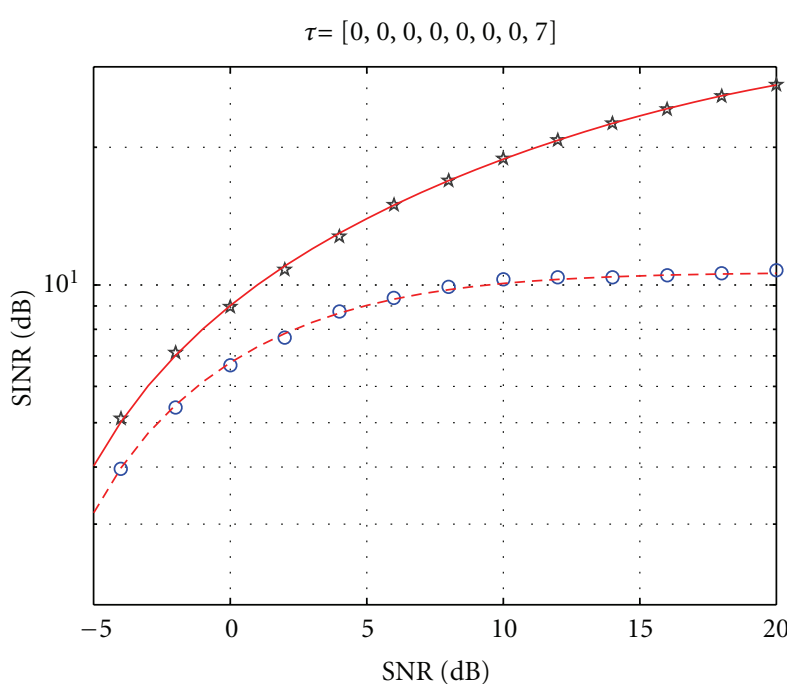

$$
\begin{aligned}
& \text { t Users 1-7, simulation } \quad \text { - Users 1-7, analysis } \\
& \text { ○ User 8, simulation }
\end{aligned}
$$

Figure 2: SINR versus SNR, $\tau_{1}=\tau_{2}=\cdots=\tau_{7}=0, \tau_{8}=7$.

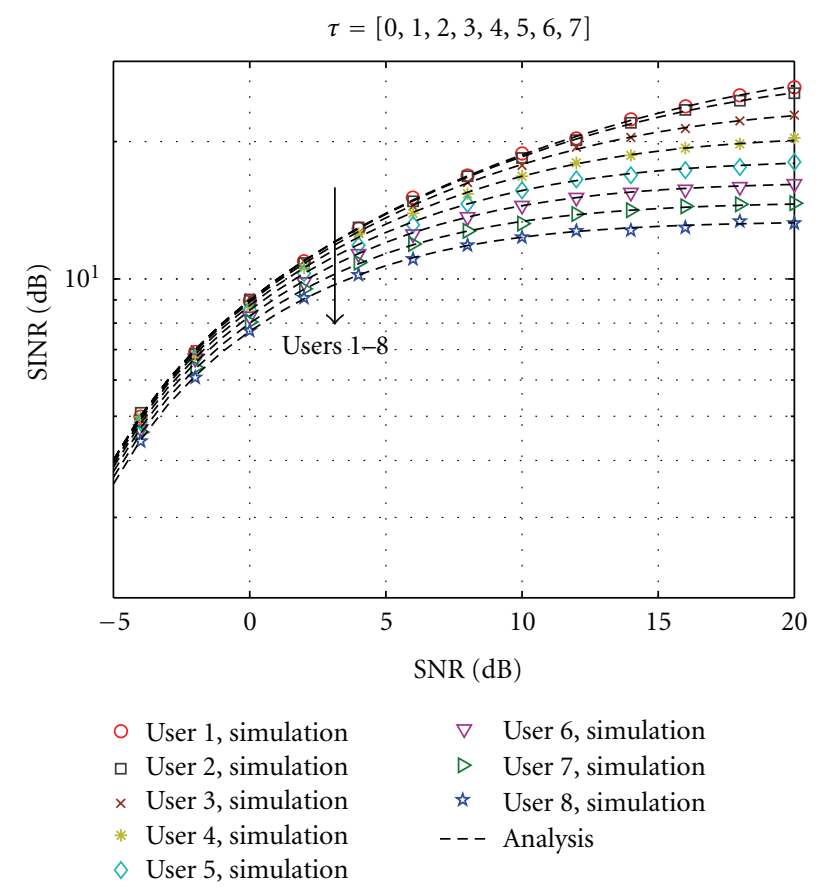

FIGURE 3: SINR versus SNR, $\tau=[0,1,2,3,4,5,6,7]$.

[4], the channel length is $L=9$, and the CP length is $L_{\mathrm{CP}}=8$. Therefore, the first asynchronous scenario considers the worst case scenario for the 8th user, because it suffers from interference caused by all the 7 previous users with a delay close to the channel memory order. Similarly, the third scenario considers the worst case scenario for the first user, where the interference comes from the rest of the 7 users with a relative delay close to the channel memory order.

It is observed from Figures 2-4 that for the three scenarios considered, the analytical results agree well with 


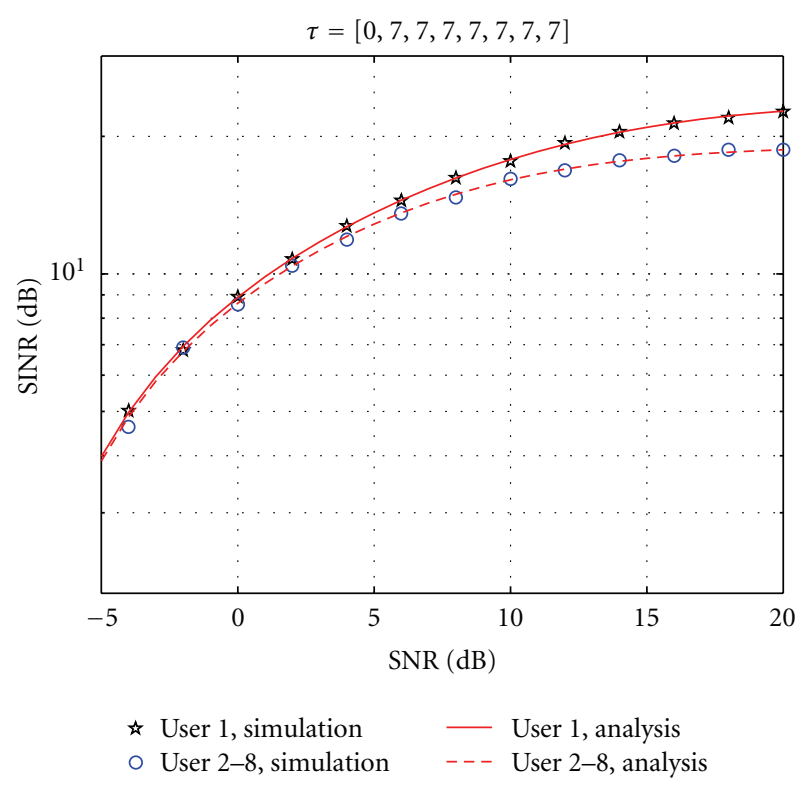

FIGURE 4: SINR versus SNR, $\tau_{1}=0, \tau_{2}=\cdots=\tau_{8}=7$.

the simulation. In addition, SINR of different users decreases with an increasing TOA. In another words, an ordering with decreasing average SINR is equivalent to ordering with increasing TOA. Applying this property of quasisynchronous BS-CDMA, we propose an iterative SIC receiver to mitigate the MUI due to quasi-synchronous reception.

\section{Iterative SIC Receiver Design}

The proposed receiver iteratively employs SIC in a blockwise manner with an ordering criterion of increasing TOA to mitigate the interference. In the first iteration, when the first user $(m=1)$ is considered, there is no interference from users whose signals arrive earlier, and $\mathbf{z}_{1}(i)$ only consists of the signals from the first user plus a noise term and a small amount of interference from the later $M-1$ users (cf. (3)). Neglecting the interference from the users whose signals arrive later for now, we have

$$
\begin{gathered}
\mathbf{w}_{1}^{(1)}(i)=\mathbf{z}_{1}(i) \approx M \tilde{\overline{\mathbf{H}}}_{1} \mathbf{s}_{1}^{(1)}(i)+\mathbf{v}(i), \\
\hat{\mathbf{s}}_{1}^{(1)}(i)=\mathbf{F}^{H} \mathbf{G}_{1} \mathbf{F} \mathbf{w}_{1}^{(1)}(i),
\end{gathered}
$$

where the notations $\hat{\mathbf{s}}_{m}^{(q)}(i)$ and $\mathbf{w}_{m}^{(q)}(i)$ are used here to denote the signals of the $m$ th user after and before equalization in the $q$ th iteration. The detected transmitted signals of the first user in the first iteration, denoted as $\overline{\mathbf{s}}_{1}^{(1)}(i)$, are then obtained by using (7), where $\widehat{\mathbf{s}}_{m}(i)$ is substituted by $\widehat{\mathbf{s}}_{1}^{(1)}(i)$.

After the signals from the first user are detected, the base station moves on to detect the signals for the second user. When the $m$ th user is considered, the signals of the first $m-1$ users in the first iteration have been obtained. These signals are used to reconstruct the $m-1$ interference terms by using
(5), where $\mathbf{x}_{b}(i)$ is replaced by $\widehat{\mathbf{x}}_{b}^{(1)}(i)$, which is the spread and precoded signal of $\overline{\mathbf{s}}_{b}^{(1)}(i)$, that is,

$$
\widehat{\mathbf{x}}_{b}^{(1)}(i)=\left(\mathbf{c}_{b} \otimes \boldsymbol{\Lambda}_{b}\right) \overline{\mathbf{s}}_{b}^{(1)}(i) .
$$

Denote the reconstructed interference terms from users whose signals arrive earlier in the first iteration as $\hat{\boldsymbol{\theta}}_{b \rightarrow m}^{(1)}(i)$. The recovered signals for the $m$ th user in the first iteration before FFT, equalization, and IFFT are given by

$$
\mathbf{w}_{m}^{(1)}(i)=\mathbf{z}_{m}(i)-\sum_{b=1}^{m-1} \hat{\boldsymbol{\theta}}_{b \rightarrow m}^{(1)}(i) .
$$

After $\mathbf{w}_{m}^{(1)}(i)$ is obtained, the transmitted symbols for the $m$ th user can be detected by following the same approach as for the first user.

So far, we have described the SIC receiver with ordering by TOA for one iteration. Note that when only one iteration is used, it is assumed that interference from users whose signals arrive later than the synchronization instant can be neglected. This assumption does not cause large performance degradations for the reference user when the interference power from users whose signals arrive later is much smaller than that from the users whose signals arrive earlier. In some cases when asynchronization is severe, interference accumulated from the signals of the users that arrive later may also cause unreliable detection of the reference user's message. Interestingly, although the detection of the symbols for some users may not be reliable due to interference from users whose signals arrive later, they do not cause much performance degradation when the erroneously detected symbols are used to reconstruct the interference terms for subsequent users in later stages of the SIC receiver. This phenomenon is verified by simulations detailed in Section 5.

After the first iteration when the signals from all users are detected, these detected signals can be used to reconstruct all interference affecting a given reference user. The interference terms from users whose signals arrive later are reconstructed by using (4), where $\mathbf{x}_{a}(i)(a=m+1, \ldots, M)$ is replaced by $\widehat{\mathbf{x}}_{a}^{(q)}(i)$, which is the spread and precoded signals of $\overline{\mathbf{s}}_{a}^{(q-1)}(i)$ from the previous iteration. Denote the reconstructed interference terms from users whose signals arrive later in the $q$ th iteration as $\hat{\phi}_{a \rightarrow m}^{(q-1)}$. The sum of these reconstructed interference terms from the later users is then subtracted to update the signals before equalization, that is,

$$
\mathbf{w}_{m}^{(q)}(i)=\mathbf{z}_{m}(i)-\sum_{b=1}^{m-1} \hat{\boldsymbol{\theta}}_{b \rightarrow m}^{(q)}(i)-\sum_{a=m+1}^{M} \hat{\boldsymbol{\phi}}_{a \rightarrow m}^{(q-1)}(i),
$$

and the transmitted symbols are detected by updating $\widehat{\mathbf{s}}_{m}^{(q)}(i)$ as

$$
\hat{\mathbf{s}}_{m}^{(q)}(i)=\mathbf{F}^{H} \mathbf{G}_{m} \mathbf{F} \mathbf{w}_{m}^{(q)}(i)
$$

followed by a log-likelihood detector.

A receiver structure of the proposed iterative SIC method is given in Figure 5, where the superscript $(\cdot)^{(q)}$ and block 


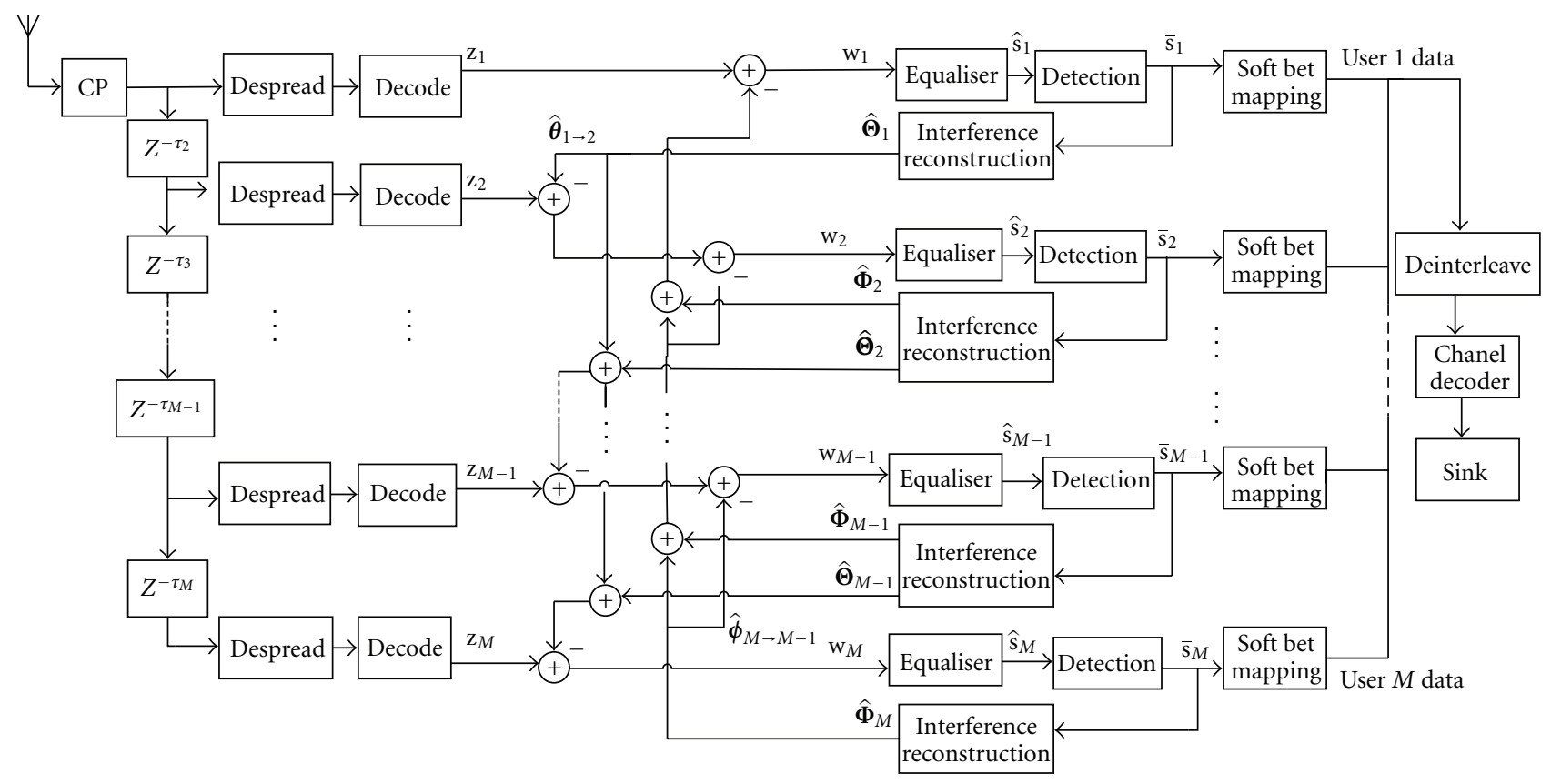

FIGURE 5: Receiver structure employing iterative SIC.

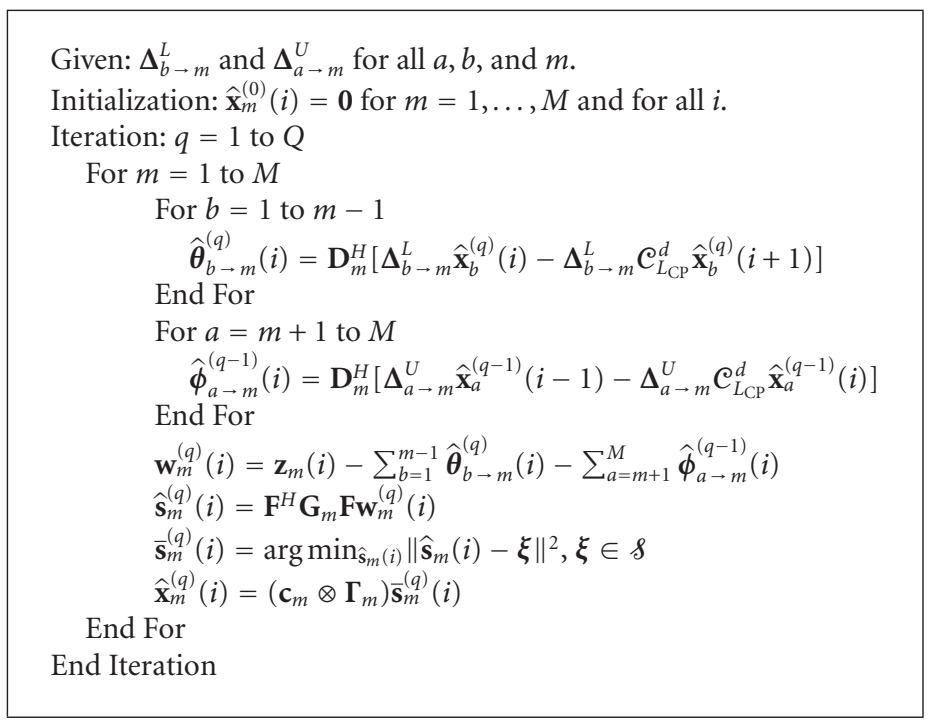

Algorithm 1: Pseudocode for detecting the signals using the iterative SIC method.

index $(\cdot)(i)$ are omitted. The notation $Z^{-\tau_{m}}$ indicates a delay of $\tau_{m}$ on the input signal. For example, when the receiver detects the signal for the second user, it needs to synchronize to the second user, which experiences a delay of $\tau_{2}$. In addition, $\hat{\boldsymbol{\Theta}}_{b}$ is used to denote an array with $\hat{\boldsymbol{\theta}}_{b \rightarrow m}$ as its $m$ th column, and $\hat{\boldsymbol{\Phi}}_{a}$ is used to denote an array with $\hat{\boldsymbol{\phi}}_{a \rightarrow m}$ as its $m$ th column. Finally, the thick and thin arrows are used to represent data flow in the form of an array or a vector, respectively.

The SIC algorithm can be employed for an arbitrary number of iterations. Our simulations in Section 5 show that simply using two iterations of SIC can provide a reasonably good performance that is close to a synchronous system. Suppose that there are $M$ users ordered and indexed according to their TOA. The pseudocode for detecting the signals using the iterative SIC method is given in Algorithm 1.

4.1. Parallel Detection to Reduce Latency. According to the SIC method presented above, to detect the signals from a user whose signals arrive later, all blocks from users whose signals arrive earlier have to be detected. For such users whose signals arrive later, large latency in their signal detection may occur. To address this issue, we propose an algorithm to control the detection order of the blocks from all users such 


\begin{tabular}{|c|c|c|c|c|c|}
\hline Bser & 1 & 2 & 3 & 4 & 5 \\
\hline 1 & & & \\
\hline 3 & & &
\end{tabular}

Figure 6: Order of detection for SIC.

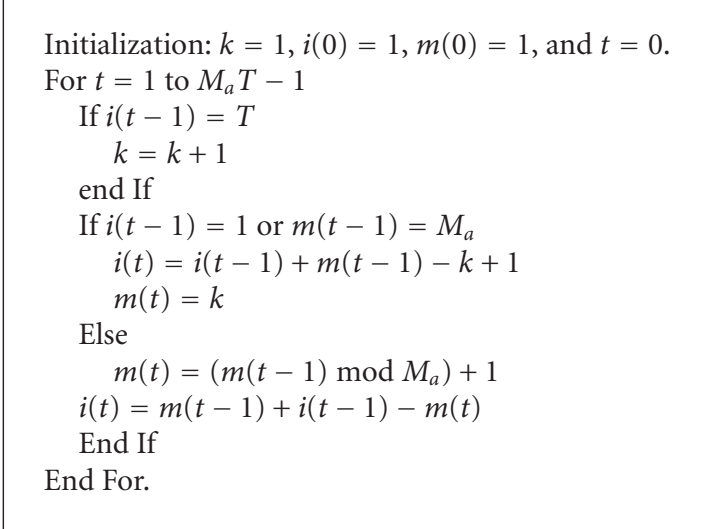

Algorithm 2: Pseudocode for determining the sequence of parallel detection of the blocks from all users in SIC.

that parallel detection of the signals from different users can be achieved to reduce the detection latency.

It is known that to detect the $i$ th block of the $m$ th user, the $i$ th and the $(i+1)$ th blocks of the first $m-1$ users need to be detected first. Consider a system with three users, each transmitting five blocks. The order of detection for blocks of all users is illustrated in Figure 6. It is observed from Figure 6 that, when there are $M_{a}$ active users, each of which transmits a total of $T$ blocks, arranging the blocks of the users in an $M_{a} \times T$ matrix where the top-left entry is the first block of the first user, the blocks are decoded sequentially by tracking the antidiagonal entries of that matrix. Alternatively, let $t$ be the time index, and let $i(t)$ and $m(t)$ denote the decoded block index and the user to which it corresponds at time $t$. The pseudocode for determining the sequence of parallel detection is given Algorithm 2, where mod denotes the moduloarithmetic function.

\section{Simulations}

We present simulation results for the quasi-synchronous BSCDMA systems using the proposed iterative SIC receiver. In all simulations, we assume perfect power control for the uplink signals. We considered quasi-synchronous BS-CDMA with QPSK modulation, a block length of $P=16$, a cyclic prefix length of $L_{\mathrm{CP}}=8$, and a channel length of 9 . We used Rayleigh fading channels with an exponentially decaying profile. The decay factor is approximately 0.86 [4]. Linear minimum mean squared error (LMMSE) frequency domain

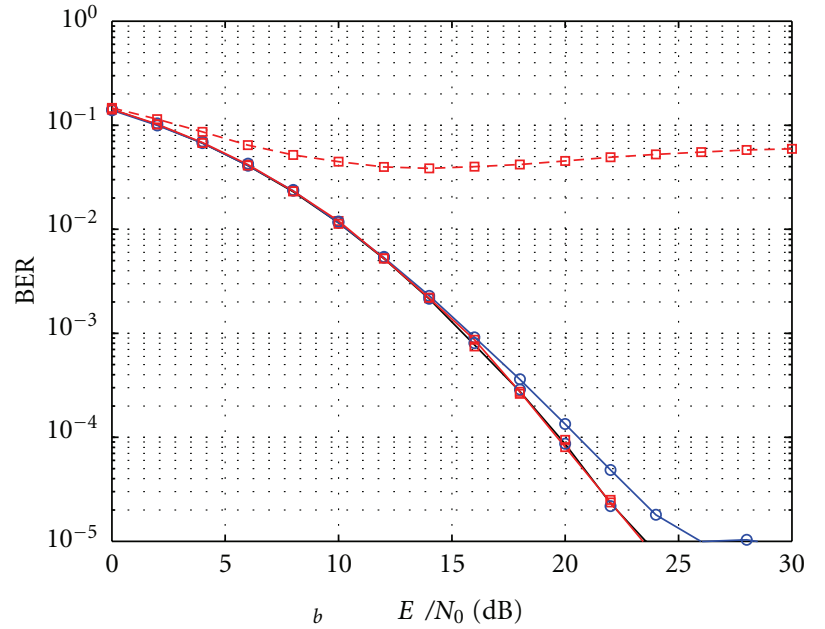

$\begin{array}{lll}- \text { Sync. RX } & \circ \text { Users 1-7, case 1, two iterations } \\ \multimap \text { Users 1-7, case 1, one iteration } \square \text { User 8, case 1, two iterations } \\ \square \text { User 8, case 1, one iteration } \quad \text { - }- \text { - User 8, case 1, without SIC }\end{array}$

FIGURE 7: BER performance of each user, $\tau_{1}=\tau_{2}=\cdots=\tau_{7}=0$, $\tau_{8}=7$.

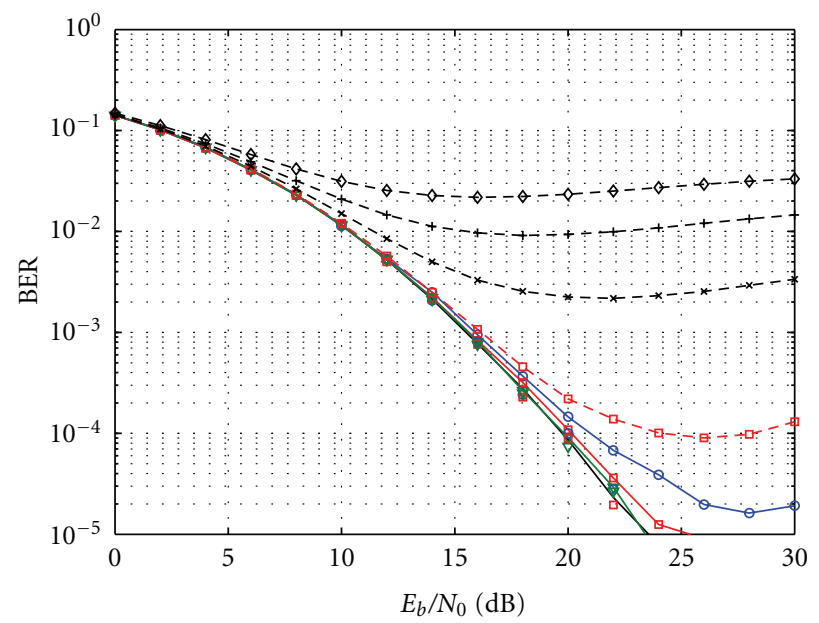

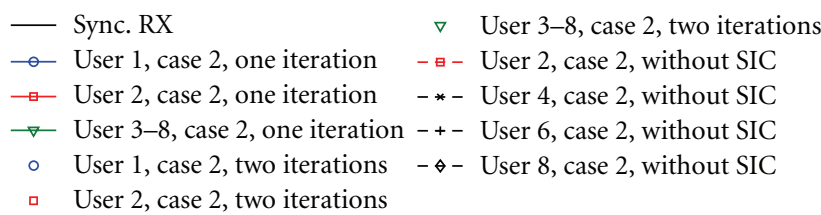

FIGURE 8: BER performance of each user, $\tau=[0,1,2,3,4,5,6,7]$.

equalizers (see $[24,28]$ ) are used to detect the transmitted symbols of all users.

We present the uncoded bit error rate (BER) performance of each user for the aforementioned quasisynchronous BS-CDMA systems using one or two iterations in Figures 7, 8, and 9, considering the three asynchronous scenarios discussed in Section 3, which are referred to as case 1 , case 2 , and case 3 , respectively. In all simulations, the curves for multiple users (e.g., users 1-7) represent the BER performance averaged over these users. The performance is 


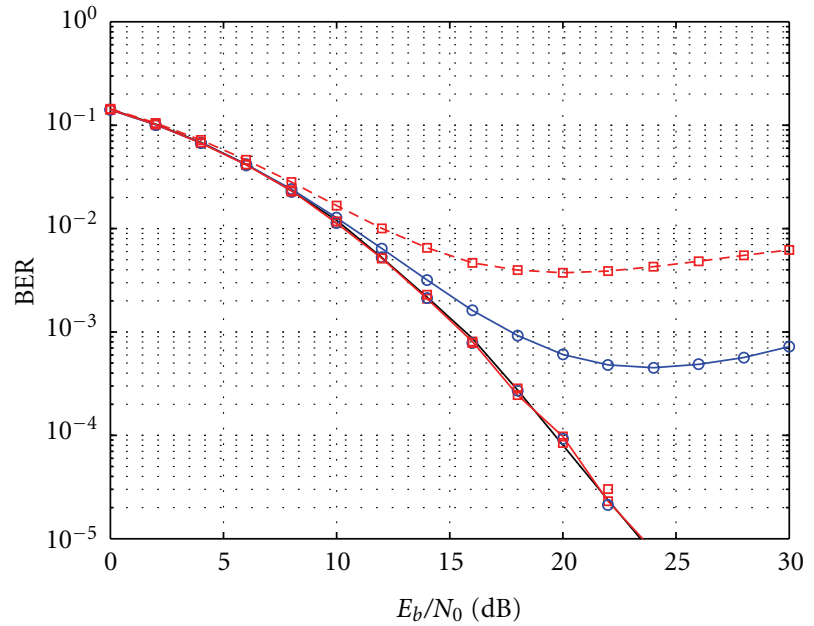

$\begin{array}{ll}- \text { Sync. } & \circ \text { User 1, case 3, two iterations } \\ \rightarrow-\text { User 1, case 3, one iteration } & \square \text { Users 2-8, case 3, two iterations } \\ \square-\text { Users 2-8, case 3, one iteration } & -\bullet-\text { Users 2-8, case 3, without SIC }\end{array}$

FIGURE 9: BER performance of each user, $\tau_{1}=0, \tau_{2}=\cdots=\tau_{8}=7$.

compared with that of a system using only one iteration, and the performance of a system that does not use SIC. Note that when the first scenario is considered, the performance of the first 7 users employing SIC with one iteration is the same as that without SIC, as SIC is only applied to the 8th user in this case. Similarly, the performance with only one iteration is the same as that without SIC for the first user in the second and third asynchronous scenarios. As a benchmark, the performance of a synchronous BS-CDMA system is also plotted.

It is observed from Figures 7-9 that for all the asynchronous scenarios considered, when SIC is not applied, a performance degradation compared to a synchronous BS-CDMA system occurs for each user, with users whose signals arrive later suffering from a more severe performance degradation due to a decreased average SINR. Comparing the performance of the 8th user without SIC in Figures 7-9 shows that the performance of the 8th user for the first scenario suffers from the most severe performance degradation due to the interference from the earlier 7 users, as opposed to the third scenario where the 8th user only suffers from interference caused by the first user.

Applying SIC for one iteration can significantly mitigate the interference from the earlier users, which is shown by the improved performance compared to that without SIC in Figures 7-9. However, it is also observed that when only one iteration is used, error floors in the performance curves of the users whose signals arrive earlier occur, due to the interference from the users whose signals arrive later. For example, an error floor of about $10^{-3}$ occurs in the performance curve of the first user for the third scenario when only one iteration is employed, since it suffers from interference from the later 7 users. Interestingly, although the detection of the earlier user's signals shows performance degradation compared to that of the synchronous system, when these erroneously detected symbols are used to reconstruct the interference

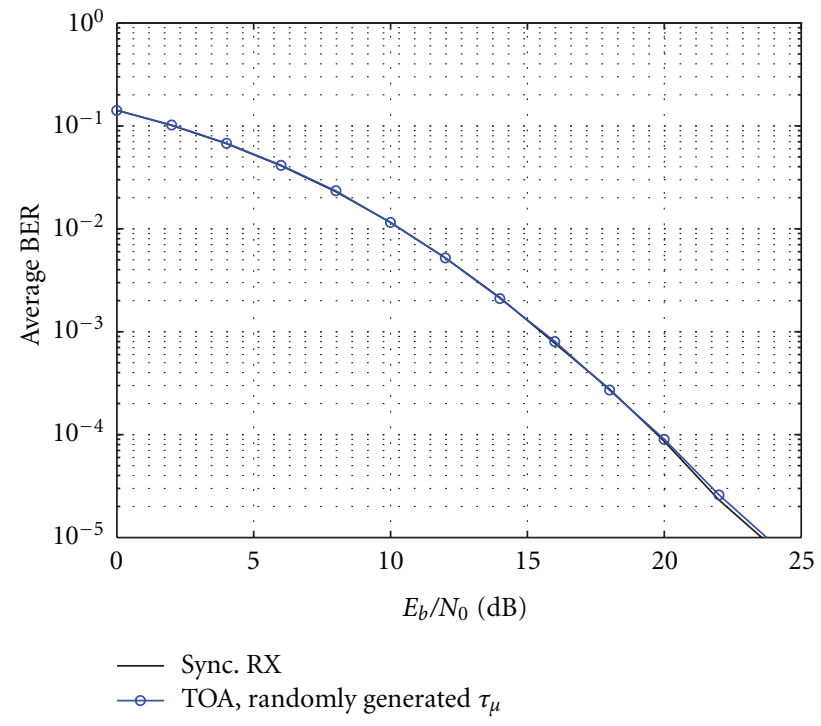

FIGURE 10: Average BER of quasi-synchronous BS-CDMA with two iteration of SIC for random uniformly distributed i.i.d. delays.

terms for the subsequent users, the performances of the subsequent users are not significantly affected. This is shown through Figures 7-9 where the performances of the later users with one iteration are almost the same as that of the synchronous systems. Note that this fact holds even when the erroneous detections of the signals for the earlier users are quite severe. For example, it is shown in Figure 9 that signal detection for the last 7 users is still reasonably reliable even though the performance of the first user shows an error floor of about $10^{-3}$. Using an additional iteration can effectively suppress the error floors in the performance curves for the earlier users. As is shown in Figures 7-9, signals from all users achieve a performance that is very close to the synchronous system when an additional iteration is applied.

The results shown in Figures 7-9 are for predetermined delays and considered the performance of individual users. In Figure 10, we show the performance of a quasi-synchronous BS-CDMA system with randomly generated delays. By using randomly generated $\tau_{\mu}$ for each channel realization, where $\tau_{\mu}$ are i.i.d. uniformly distributed integers and $\tau_{\mu} \in$ $\left(0, \max \left\{\tau_{\mu}\right\}\right)$, the average BER for quasi-synchronous BSCDMA is obtained by taking the average of the BERs over all channel realizations and all users. It is observed that when the delays are the i.i.d. uniformly distributed random variables, a quasi-synchronous BS-CDMA system still achieves nearly the same performance as that of a synchronous system when two iterations of the proposed SIC receiver is used.

Figure 11 shows the BER performance averaged over 8 users for the systems considered above. In addition, the performances of quasi-synchronous BS-CDMA systems where instantaneous received signal power of each user is used as the ordering criterion is also shown. With such a criterion, users are ordered and indexed according to the received power of their signals, where users' signals with the largest receive power are first detected. These detected signals are used to reconstruct the interference, which is 


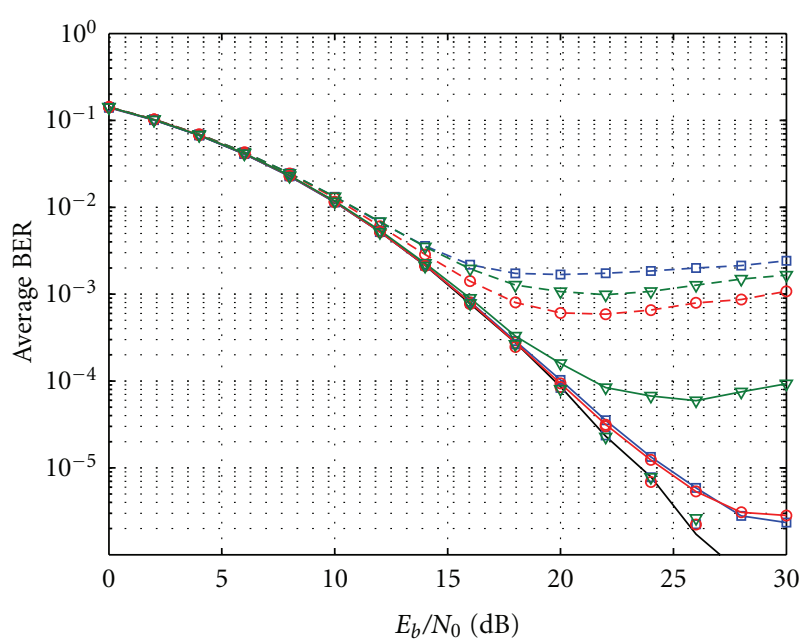

- Sync. RX

$-\because-$ Received power, case 1

$-\theta-$ Received power, case 2

$-\nabla-$ Received power, case 3

$\rightarrow$ TOA, case 1, one iteration

$$
\begin{array}{cl}
- & \text { TOA, case } 2 \text {, one iteration } \\
\rightarrow & \text { TOA, case } 3 \text {, one iteration } \\
\square & \text { TOA, case 1, two iterations } \\
\circ & \text { TOA, case 2, two iterations } \\
\nabla & \text { TOA, case 3, two iterations }
\end{array}
$$

FIGURE 11: Average BER performance of quasi-synchronous BSCDMA with 8 active users.

subsequently removed when detecting the signals of the user with the second largest received power. It is shown that conventional SIC using instantaneous received signal power as the ordering criterion suffers from a performance degradation in quasi-synchronous BS-CDMA. However, using SIC with ordering based on TOA significantly mitigates the error floors after one iteration and achieves a performance close to that of a synchronous system with two iterations.

\section{Conclusions}

This paper proposed using the increasing TOA of different users as the ordering criterion for an iterative SIC receiver in quasi-synchronous BS-CDMA systems. The ordering criterion is based on analysis and simulation of the average SINRs of different users, where it was shown that an increasing order of TOA is essentially equivalent to a decreasing order of average SINR in such systems operating in exponentially decaying channels. In addition, an algorithm that determines the detection order of different blocks to allow parallel detection of the signals from different users is also proposed such that minimal latency in detection occurs. Simulation results showing the performance of the proposed iterative SIC receiver in a quasi-synchronous BS-CDMA system demonstrated that using two iterations in the SIC receiver is sufficient to provide a performance close to a synchronous system.

\section{Acknowledgments}

The authors would like to thank Dr. Filippo Tosato and Dr. Rafael Cepeda for the discussions on the channel model and providing the references. The authors would also like to thank the directors at TRL for their continued support.

\section{References}

[1] J. P. Coon, “Generalized precoded block-spread CDMA," IEEE Transactions on Communications, vol. 57, no. 7, pp. 19191923, 2009.

[2] "Physcial layer aspect for evolved UTRA," 3GPP TR 25.814 V7.1.0, 3rd Generation Partnership Project Std., September 2006.

[3] X. Tang and W. H. Mow, "Design of spreading codes for quasisynchronous CDMA with intercell interference," IEEE Journal on Selected Areas in Communications, vol. 24, no. 1, pp. 84-93, 2006.

[4] M. Z. Bocus, Y. Wang, and J. P. Coon, "Linear equalizers for quasi-synchronous block spreading CDMA systems," in Proceedings of the IEEE Global Telecommunications Conference (GLOBECOM '09), pp. 1-6, December 2009.

[5] J. G. Proakis, Digital Communications, McGraw-Hill, New York, NY, USA, 4th edition, 2001.

[6] M. Mohammad and R. Michael Buehrer, "The effects of ordering criteria in linear successive interference cancellation in CDMA systems," IEEE Transactions on Wireless Communications, vol. 7, no. 11, Article ID 4684589, pp. 4128-4132, 2008.

[7] J. G. Andrews, "Interference cancellation for cellular systems: a contemporary overview," IEEE Wireless Communications, vol. 12, no. 2, pp. 19-29, 2005.

[8] R. Michael Buehrer, "Equal BER performance in linear successive interference cancellation for CDMA systems," IEEE Transactions on Communications, vol. 49, no. 7, pp. 12501258, 2001.

[9] W. T. Sang, K. B. Letaief, and R. S. Cheng, "Combined coding and successive interference cancellation with random interleaving for DS/CDMA communications," in Proceedings of the 50thVehicular Technology Conference (VTC-Fall '99), pp. 1825-1829, 1999.

[10] H. Nie, P. T. Mathiopoulos, and G. K. Karagiannidis, "Reverse link capacity analysis of cellular CDMA systems with controlled power disparities and successive interference cancellation," IEEE Transactions on Wireless Communications, vol. 5, no. 9, Article ID 1687768, pp. 2447-2456, 2006.

[11] P. Patel and J. Holtzman, "Analysis of a simple successive interference cancellation scheme in a DS/CDMA system," IEEE Journal on Selected Areas in Communications, vol. 12, no. 5, pp. 796-807, 1994.

[12] Y. Ma, P. H. Tan, and T. J. Lim, "Systematic approach to multistage linear successive interference cancellation for multiuser detection in dynamic asynchronous CDMA systems," Electronics Letters, vol. 35, no. 3, pp. 208-209, 1999.

[13] X. Ren, S. Zhou, Y. Yao, and Z. Zhou, "A new successive interference cancellation for asynchronous CDMA," in Proceedings of the IEEE Global Telecommunications Conference (GLOBECOM '03), vol. 1, pp. 252-256, December 2003.

[14] C. Lee, K. Sohraby, and K. Kim, "Performance of successive interference cancellation scheme considering the power control step size in DS/CDMA systems," in Proceedings of the IEEE 60th Vehicular Technology Conference (VTC2004-Fall '04), pp. 779-783, September 2004.

[15] K. S. Gilhousen, I. M. Jacobs, R. Padovani, A. J. Viterbi, L. A. Weaver, and C. E. Wheatley, "On the capacity of a cellular CDMA system," IEEE Transactions on Vehicular Technology, vol. 40, no. 2, pp. 303-312, 1991.

[16] S. A. Jafar, "The ergodic capacity of interference networks," http://arxiv.org/abs/0902.0838. 
[17] K. Balachandran, S. R. Kadaba, and S. Nanda, "Channel quality estimation and rate adaptation for cellular mobile radio," IEEE Journal on Selected Areas in Communications, vol. 17, no. 7, pp. 1244-1256, 1999.

[18] X. Peng, T. S. Dharma, F. Chin, and A. S. Madhukumar, "Novel interference cancellation methods for BS-CDMA in uplink broadband mobile communication systems," IEEE Communications Letters, vol. 12, no. 8, pp. 560-562, 2008.

[19] J. Laurila, A. F. Molisch, and E. Bonek, "Influence of the scatterer distribution on power delay profiles andazimuthal power spectra of mobile radio channels," in Proceedings of the IEEE 5th International Symposium on Spread Spectrum Techniques and Applications, vol. 1, pp. 267-271, September 1998.

[20] "Spatial channel model for Multiple Input Multiple Output (MIMO) simulations (Release 8)," 3GPP TR 25.996 V8.0.0, 3rd Generation Partnership Project Std., December 2008.

[21] H. Koorapaty, "Cramer-Rao bounds for time of arrival estimation in cellular systems," in Proceedings of the IEEE 59th Vehicular Technology Conference (VTC-Spring '04), vol. 5, pp. 2729-2733, May 2004.

[22] A. Lee Swindlehurst, "Time Delay and Spatial Signature Estimation Using Known Asynchronous Signals," IEEE Transactions on Signal Processing, vol. 46, no. 2, pp. 449-462, 1998.

[23] A. L. C. Hui and K. B. Letaief, "Successive interference cancellation for multiuser asynchronous DS/CDMA detectors in multipath fading links," IEEE Transactions on Communications, vol. 46, no. 3, pp. 384-391, 1998.

[24] H. Sari, G. Karam, and I. Jeanclaude, "Transmission techniques for digital terrestrial TV broadcasting," IEEE Communications Magazine, vol. 33, no. 2, pp. 100-109, 1995.

[25] D. Falconer, S. L. Ariyavisitakul, A. Benyamin-Seeyar, and B. Eidson, "White paper: frequency domain equalization for single-carrier broadband wireless systems," Tech. Rep., February 2002, http://www.sce.carleton.ca/bbw/papers/whitepaper2.pdf.

[26] Z. Wang and G. B. Giannakis, "Wireless multicarrier communications: Where Fourier meets Shannon," IEEE Signal Processing Magazine, vol. 17, no. 3, pp. 29-48, 2000.

[27] V. Erceg, D. G. Michelson, S. S. Ghassemzadeh et al., "Model for the multipath delay profile of fixed wireless channels," IEEE Journal on Selected Areas in Communications, vol. 17, no. 3, pp. 399-410, 1999.

[28] D. Falconer, S. L. Ariyavisitakul, A. Benyamin-Seeyar, and B. Eidson, "Frequency domain equalization for single-carrier broadband wireless systems," IEEE Communications Magazine, vol. 40, no. 4, pp. 58-66, 2002. 